\title{
Lattice polygons and families of curves on rational surfaces
}

\author{
Niels Lubbes · Josef Schicho
}

Received: 12 February 2010 / Accepted: 14 November 2010 / Published online: 4 December 2010

(C) Springer Science+Business Media, LLC 2010

\begin{abstract}
First we solve the problem of finding minimal degree families on toric surfaces by reducing it to lattice geometry. Then we describe how to find minimal degree families on, more generally, rational complex projective surfaces.
\end{abstract}

Keywords Algebraic geometry · Toric geometry · Lattice polygons · Families of curves $\cdot$ Surfaces

\section{Introduction}

Every algebraic surface in projective space $\mathbf{P}^{r}$ can be generated by a family of curves in projective space (e.g. the hyperplane sections). For a fixed surface, this can be done in infinitely many ways. Maybe the simplest family of algebraic curves is one where the curves have minimal genus, and among those one with minimal degree. In this paper, we study the families of genus zero curves of minimal degree, in the case where the given surface is rational (Sect. 8). Classical examples of such families are the families of lines on a ruled surface - with the single example of the nonsingular quadric in $\mathbf{P}^{3}$ having two such families - and the families of conics on a non-ruled conical surface (the surfaces with more than one family of conics have been classified in [11]).

The paper starts with a seemingly quite different topic, namely the study of discrete directions which minimize the width of a given convex lattice polytope (Sect. 2). As the lattice points remind of sticks in a vineyard, we call the problem of finding all these directions the "vineyard problem"; for the minimal directions, most sticks are

\footnotetext{
N. Lubbes $(\bowtie) \cdot$ J. Schicho

Johann Radon Institut, Österreichische Akademie der Wissenschaften, Linz, Austria

e-mail: niels.lubbes@oeaw.ac.at

J. Schicho

e-mail: josef.schicho@oeaw.ac.at
} 
aligned with others and one "sees" only a minimal number. We give an elementary solution, based on the notion of the adjoint lattice polytope, which is defined as the convex hull of the interior lattice points (see Sect. 3).

The vineyard problem is equivalent to the specialization of the problem of finding toric families of minimal degree on a given toric surface (see Proposition 31). The main result of this paper is the fact that our elementary solution can be translated into the language of toric geometry, and then generalizes in a natural way so that it makes it possible to construct all minimal degree families of rational curves on arbitrary rational surfaces! In Sect. 7, we give a proof in the language of algebraic geometry (which subsumes then the elementary proof in Sect. 3). The methods are quite different, but, as the reader may check, there is a close analogy in the structure of the two proofs.

The algebraic geometry analogue of the adjoint lattice polytope is adjunction; this has been observed in [4] (see also [5, 12]).

\subsection{Overview}

The following table gives the problems and their solutions which are treated in this document:

\begin{tabular}{lll}
\hline Problem & Solution & Description \\
\hline Definition 7 & Theorem 13 & Vineyard problem (or viewangle problem on vineyards) \\
Definition 28 & Proposition 31 & Toric family problem on toric surfaces \\
Definition 41 & Theorem 46 & Rational family problem on polarized rational surfaces \\
Definition 49 & Proposition 50 & Rational family problem on rational surfaces \\
\hline
\end{tabular}

The second problem is reduced to the first problem and the fourth problem is reduced to the third problem. In Sect. 2 we define convex lattice polygons and their adjoints. In Sect. 4 we will define what we mean by family and give properties, of which Proposition 20 is most important. For Sect. 5 only Definition 16 is needed of Sect. 4. In Sect. 6 we summarize the notions of minimally polarized rational surface (mprs for short), adjoint relation and adjoint chain, which are used in Sect. 7. See Remark 47 for the analogy between Sects. 3 and 7.

\subsection{Guide for reading}

We explain the structure of this document. The main claims are labeled by ' $[\mathbf{a}-\mathbf{z}])$ '. A claim is given by the sentence starting with 'Claim [1-10]:' and is a step for proving the main claims. The proof of a claim is given by the remaining sentences in the same paragraph. We define each sentence in the proof of a claim to be a sub-claim.

\section{Convex lattice polygons}

Definition 1 (Lattice and dual lattice) A lattice $\Lambda_{n}$ is defined as $\mathbf{Z}^{n} \subset \mathbf{R}^{n}$. Its dual lattice $\Lambda_{n}^{*}$ is defined as $\operatorname{Hom}_{\mathbf{Z}}\left(\Lambda_{n}, \mathbf{Z}\right)$. A lattice equivalence is a map (translation, 


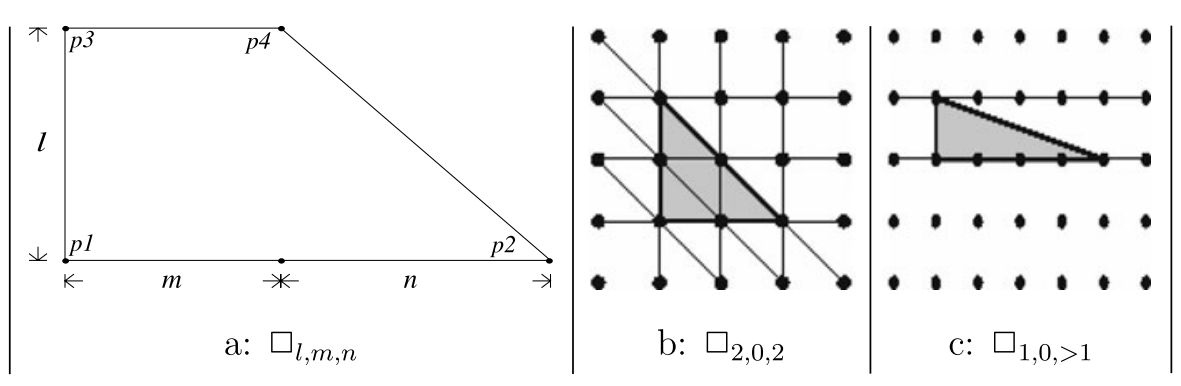

Fig. 1 Convex lattice polytopes corresponding to $\square_{l, m, n}$

rotation, shearing and reflection):

$$
\Phi: \mathbf{R}^{n} \rightarrow \mathbf{R}^{n}, \quad \vec{x} \mapsto A \vec{x}+\vec{y},
$$

where $A \in G L_{n}(\mathbf{Z})$ and $\vec{y} \in \mathbf{Z}^{n}$. We will denote $\Lambda_{2}$ by $\Lambda$.

Definition 2 (Convex lattice polygon) Let $\Lambda$ be a two-dimensional lattice. A convex lattice polygon $\Gamma$ is the convex hull of a finite non-empty set of lattice points in $\Lambda$. Polygons are considered equivalent when they are lattice equivalent.

Definition 3 (Attributes of polygons) Let $\Gamma$ be a lattice polygon with lattice $\Lambda$. We call $\Gamma$ a shoe polygon if and only if $\Gamma=\square_{l, m, n}$ where

$$
\square_{l, m, n}:=\text { ConvexHull}((0,0),(0, l),(m, l),(m+n, 0)),
$$

where $l, m, n \in \mathbf{Z}_{\geq 0}$ (see Fig. 1a)). We call $\Gamma$ a standard triangle if and only if $\Gamma=\square_{l, 0, l}$ with $l>0$ (for example Fig. 1b). We call $\Gamma$ a thin triangle if and only if $\Gamma=\square_{1,0, l}$ with $l>1$ (for example Fig. 1c). We call $\Gamma$ minimal if and only if $\Gamma$ is not a point and either $\Gamma$ has one interior lattice point or $\Gamma$ has no interior lattice points.

Definition 4 (Adjoint polygon) Let $\Gamma$ be a convex lattice polygon with lattice $\Lambda$. The adjoint polygon $\Gamma^{\prime}$ of $\Gamma$ is defined as the convex hull of the interior lattice points of $\Gamma$ (if there exist any). We denote the adjoint of $\Gamma$ taken $i$ times by $\Gamma^{i}$.

Definition 5 (Viewangles and width) Let $\Gamma$ be a convex lattice polygon with lattice $\Lambda$. A viewangle for $\Gamma$ is a nonzero vector $h \in \Lambda^{*}-\{0\}$ in the dual lattice. The viewangle width of a viewangle $h$ for $\Gamma$ is

$$
\text { width }_{\Gamma}: \Lambda^{*} \rightarrow \mathbf{Z}, \quad h \mapsto \max _{v \in \Gamma} h(v)-\min _{w \in \Gamma} h(w)
$$

The width of a convex lattice polygon is the smallest possible viewangle width:

$$
v(\Gamma)=\min _{h \in \Lambda^{*}-0} \operatorname{width}_{\Gamma}(h) .
$$


Fig. 2 A convex lattice polygon and two viewangles.

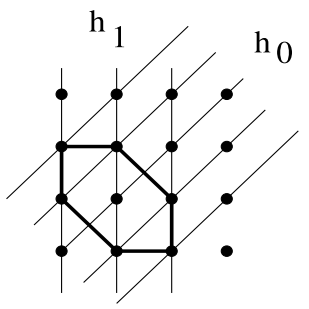

The set of optimal viewangles on $\Gamma$ is defined as

$$
S(\Gamma)=\left\{h \in \Lambda^{*}-\{0\} \mid \operatorname{width}_{\Gamma}(h)=v(\Gamma)\right\} .
$$

Definition 6 (Attributes of viewangles) Let $\Gamma$ be a convex lattice polygon with lattice $\Lambda$. Let $h \in \Lambda^{*}-\{0\}$ be a viewangle. Tight viewangles: We call $h$ max-tight for $\Gamma$ if and only if $\Gamma^{\prime}$ is defined and $\max _{v \in \Gamma} h(v)=\max _{w \in \Gamma^{\prime}} h(w)+1$. We call $h$ mintight for $\Gamma$ if and only if $\Gamma^{\prime}$ is defined and $\min _{v \in \Gamma} h(v)=\min _{w \in \Gamma^{\prime}} h(w)-1$. We call $h$ tight for $\Gamma$ if and only if $h$ is max-tight and min-tight for $\Gamma$. Edge viewangles: We call $h$ a max-edge for $\Gamma$ if and only if $h(v)=h(w)=\max _{u \in \Gamma} h(u)$ for some $v, w \in \Gamma$ where $v \neq w$. We call $h$ a min-edge for $\Gamma$ if and only if $h(v)=h(w)=\min _{u \in \Gamma} h(u)$ for some $v, w \in \Gamma$ where $v \neq w$. We call $h$ an edge for $\Gamma$ if and only if $h$ is a maxedge and min-edge for $\Gamma$.

\section{Minimal width viewangles for convex lattice polygons}

Definition 7 (Vineyard problem) Given a convex lattice polygon $\Gamma$ find the width $v(\Gamma)$ and all optimal viewangles $S(\Gamma)$ (see Definition 5).

Example 8 (Vineyard problem) Let $\Gamma$ be the convex lattice polygon as in Fig. 2 with viewangles $h_{0}=(1,-1)$ and $h_{1}=(1,0)$. The origin is defined by the interior lattice point of $\Gamma$.

We have width ${ }_{\Gamma}\left(h_{0}\right)=4$ and width $_{\Gamma}\left(h_{1}\right)=2$. We find for this easy example that $v(\Gamma)=2$. The optimal viewangles are $h_{1}$, the horizontal viewangle $(0,1)$ and the diagonal viewangle $(-1,-1)$.

Lemma 9 (Lowerbound) Let $\Gamma$ be a convex lattice polygon which is not minimal (see Definition 3 for minimal) with lattice $\Lambda$. Let $h \in \Lambda^{*}-\{0\}$ be a viewangle.

We have width $_{\Gamma}(h) \geq$ width $_{\Gamma^{\prime}}(h)+2$, and equality holds if and only if $h$ is tight for $\Gamma$.

Proof Direct consequence of Definitions 5 and 6.

Lemma 10 (Tight) Let $\Gamma$ be a convex lattice polygon which is not minimal (see Definition 3 for minimal) with lattice $\Lambda$. Let $h \in \Lambda^{*}-\{0\}$ be a viewangle. 
Fig. 3 Proof of Lemma 10

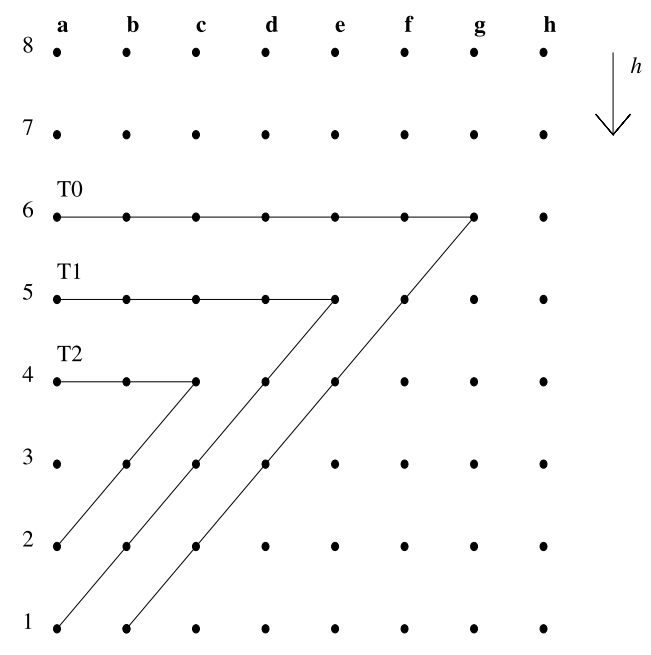

(a) If $h$ is an edge of $\Gamma^{\prime}$ then $h$ is tight for $\Gamma$.

(b) If $h$ is tight for $\Gamma^{\prime}$ then $h$ is tight for $\Gamma$.

Proof We assume that $h$ is not max-tight for $\Gamma$ in the remainder of the proof. Let $p \in \Gamma$ be such that $\max _{v \in \Gamma} h(v)=h(p)$. We will denote the lattice points in Fig. 3 by the checkboard coordinates $\mathbf{a 8}$ until h1.

Claim 1: We may assume without loss of generality that $h=(1,0), h(\mathbf{e 5})=$ $\max _{v \in \Gamma^{\prime}} h(v)$ and $p$ is right of column $\mathbf{f}$. From the assumption that $h$ is not maxtight it follows that $p$ is right of column of $\mathbf{f}$.

Let $S=\Gamma \cap L$ be a line segment where $L$ is the line corresponding to column $\mathbf{f}$.

Claim 2: The line segment $S$ does not contain interior lattice points and is not empty. Suppose by contradiction that $S$ contains an interior lattice point $q$. Then $q \in \Gamma^{\prime}$ and $h(q)>h(\mathbf{e 5})$. \&

Claim 3: We may assume without loss of generality that f6 and $\mathbf{f 5}$ are the lattice points above respectively under $S$. From Claim 2 it follows that $S$ is between $\mathbf{f i}+\mathbf{1}$ and $\mathbf{f i}$ for some $i \in \mathbf{Z}$. We apply shearing such that $\mathbf{f 6}$ and $\mathbf{f 5}$ are the required points. We have that $h$ remains unchanged under the corresponding dual transformation.

Let $Q=$ ConvexHull(f6, f5, $\Gamma \cap$ the area right of column $\mathbf{f})$.

Claim 4: The polygon $Q$ does not contain interior lattice points and is not empty. It follows from the assumption that $h$ is not max-tight.

For example $Q$ is ConvexHull(f6, f5, g7, h7) or ConvexHull(f6, f5, h6). For constructing examples it is required that $Q$ does not contain interior lattice points and that $\mathbf{e 5}$ is between the line through $(\mathbf{f 6}, \mathrm{p})$ and the line through $(\mathbf{f 5}, \mathrm{p})$. Let $\hat{\Gamma}=\operatorname{ConvexHull}(\Gamma-Q, \mathbf{g 6})$. Let $T_{0}, T_{1}$ and $T_{2}$ be the area contained by the corresponding line as in Fig. 3.

Claim 5: We have $\hat{\Gamma} \subseteq T_{0}, \Gamma^{\prime} \subseteq T_{1}$ and $\Gamma^{\prime \prime} \subseteq T_{2}$. Suppose by contradiction that $\hat{\Gamma}$ has a point outside of $T_{0}$. It follows that $\Gamma$ is not convex. $\measuredangle$ We have $\Gamma^{\prime} \subseteq \hat{\Gamma}^{\prime} \subseteq$ $T_{0}^{\prime}=T_{1}$ and $\Gamma^{\prime \prime} \subseteq T_{1}^{\prime}=T_{2}$.

Claim 6: If $h$ is not max-tight for $\Gamma$ then $h$ is not a max-edge of $\Gamma^{\prime}$. From Claim 5 and Fig. 3 it follows that $h$ reaches the maximum only once for $\Gamma^{\prime} \subseteq T_{1}$ at e5. It follows that $h$ is not a max-edge for $\Gamma^{\prime}$. 
Claim 7: If $h$ is not max-tight for $\Gamma$ then $h$ is not max-tight for $\Gamma^{\prime}$. From Claim 5 and Fig. 3 it follows that $h$ reaches a maximum for $\Gamma^{\prime \prime} \subseteq T_{2}$ on or left of column c. It follows that $h$ is not max-tight for $\Gamma^{\prime}$.

Claim 8: From Claim 6 and Claim 7 it follows that (a) and (b). The proof of Claim 6 and Claim 7 for min-edge and min-tight is completely symmetric. The statements are dual to (a) and (b).

Proposition 11 (Classification of optimal viewangles for minimal convex lattice polygons)

(a) All the optimal viewangles on minimal convex lattice polygons are classified in Fig. 4.

(b) If $\Gamma$ has a thin triangle (id est Fig. 4(20)) as adjoint then $S(\Gamma)=S\left(\Gamma^{\prime}\right)$ and the optimal viewangle is tight for $\Gamma$.

Proof The classification of minimal convex lattice polygons (see Definition 3) can be found in [12]. The classification of the optimal viewangles in Fig. 4 is a direct result of tedious case by case inspection. Let us assume $\Gamma$ is a convex lattice polygon such that $\Gamma^{\prime}=\square_{1,0, l}$ and $l>1$ (id est thin triangle).

Claim: We have $l=2$ and the optimal direction of $\Gamma$ is tight. If $l>2$ then $\Gamma$ is not convex. There is a finite number of possibilities for $\Gamma$, and for each of them the optimal direction is tight.

Definition 12 (Case distinction) Let $\Gamma$ be a convex lattice polygon with lattice $\Lambda$. We distinguish between the following cases where $\Gamma$ is not minimal except at A0:

\begin{tabular}{lll}
\hline & $\Gamma$ & $\Gamma^{\prime}$ \\
\hline A0 & Minimal & Point or emptyset \\
A1 & Standard triangle & Standard triangle \\
A2 & Not standard triangle & Standard triangle \\
A3 & Not standard triangle & Minimal and not standard triangle \\
A4 & Not standard triangle & Not minimal and not standard triangle \\
\hline
\end{tabular}

See Definition 3 for the notion of standard triangle.

Theorem 13 (Optimal viewangles) Let $\Gamma$ be a convex lattice polygon with lattice $\Lambda$. Let $S(\Gamma)$ be the set of all optimal viewangles of $\Gamma$. Let A0 until A4 be as in Definition 12 .

(a) If $A 0$ then $S(\Gamma)$ and $v(\Gamma)$ are as classified in Fig. 4.

(b) If A1 then $S(\Gamma)$ contains exactly its three edges and $v(\Gamma)=v\left(\Gamma^{\prime}\right)+3$.

(c) If A2 then $S(\Gamma)=\left\{h \in S\left(\Gamma^{\prime}\right) \mid h\right.$ tight for $\left.\Gamma\right\}$ and $v(\Gamma)=v\left(\Gamma^{\prime}\right)+2$.

(d) If $A 3$ or $A 4$ then $S(\Gamma)=S\left(\Gamma^{\prime}\right)$ and $v(\Gamma)=v\left(\Gamma^{\prime}\right)+2$. 


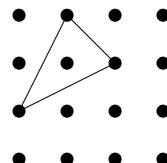

1: $(2,3)$ tight

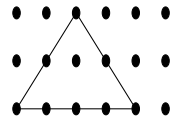

5: $(2,1)$ tight

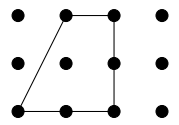

9: $(2,2)$ tight

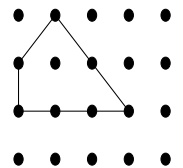

13: $(2,1)$ tight

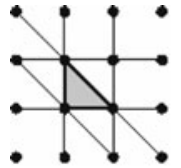

17: $(1,3) \triangle_{1}$

.....

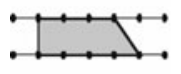

.....

$\cdots \cdots$

21: $(1,1)$ edge

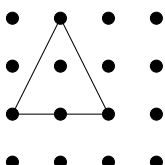

2: $(2,2)$ tight

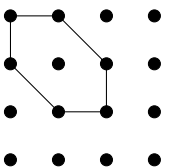

6: $(2,3)$ tight

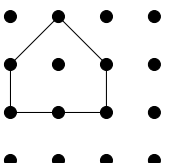

10: $(2,2)$ tight

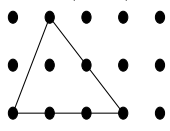

14: $(2,1)$ tight

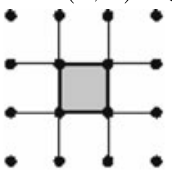

15: $(2,2)$ tight

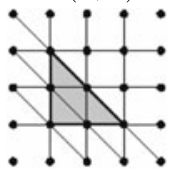

16: $(2,4)$ tight

18: $(1,2)$ edge

19: $(2,3) \triangle_{2}$

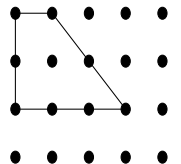

4: $(2,1)$ tight

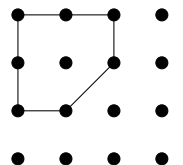

8: $(2,2)$ tight

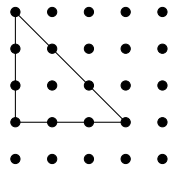

12: $(3,3) \triangle_{4}$

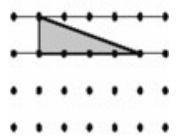

20: $(1,1)$

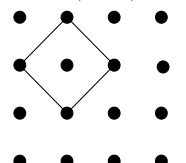

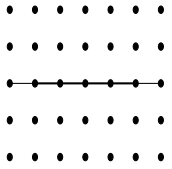

22: $(0,1)$ edge

Fig. 4 All the optimal viewangles for minimal convex lattice polygons and $(v(\Gamma)$,\#S) where \#S is the number of optimal viewangles. We denote standard triangles of length $i$ by $\triangle_{i}$

Proof We have that (a) and (b) are a direct consequence of Proposition 11 and the definition of the standard triangle. Let $T(\Gamma)=\{h \mid h \in S(\Gamma)$ and $h$ is tight $\}$.

Claim 1: If $T\left(\Gamma^{\prime}\right) \neq \emptyset$ then $S(\Gamma)=T(\Gamma)$. From Lemma 9 and Lemma 10(a) it follows that if $h \in T\left(\Gamma^{\prime}\right)$ then $\operatorname{width}_{\Gamma}(h)=v\left(\Gamma^{\prime}\right)+2$. From Lemma 9 it follows that if $h \in S(\Gamma)$ then width $\Gamma(h) \geq v\left(\Gamma^{\prime}\right)+2$ and equality holds if and only if $h \in T(\Gamma)$.

Claim 2: If $S\left(\Gamma^{\prime}\right)=T\left(\Gamma^{\prime}\right)$ then $S(\Gamma)=S\left(\Gamma^{\prime}\right)$. From Lemma 9 and Lemma 10(a) it follows that if $h \in T\left(\Gamma^{\prime}\right)$ then width $_{\Gamma}(h)=v\left(\Gamma^{\prime}\right)+2$. It follows that $T(\Gamma) \supseteq$ $T\left(\Gamma^{\prime}\right)$. If $h \in T(\Gamma)$ then width $_{\Gamma}(h)=$ width $_{\Gamma^{\prime}}(h)+2$ and thus $h \in S\left(\Gamma^{\prime}\right)$. It follows 
Fig. 5 The outer convex lattice polygon without $p_{1}$ is not a standard triangle, and its adjoint is a standard triangle

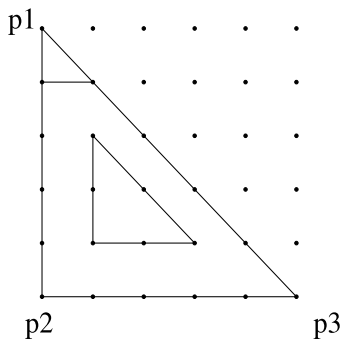

that $T(\Gamma) \subseteq S\left(\Gamma^{\prime}\right)$. From Claim 2 and the assumption it follows that $S(\Gamma)=T(\Gamma)$ and $S\left(\Gamma^{\prime}\right)=T\left(\Gamma^{\prime}\right)$.

In Fig. 5 the adjoint convex lattice polygon is a standard triangle of length 2 . The cornerpoints are denoted $p_{1}, p_{2}$ and $p_{3}$.

Claim 3: If $\mathrm{A} 2(\Gamma)$ then $S(\Gamma)=T(\Gamma)=\left\{h \in S\left(\Gamma^{\prime}\right) \mid h\right.$ tight for $\left.\Gamma\right\}$. From Lemma 9 it follows that $\Gamma \subset$ ConvexHull $\left(p_{1}, p_{2}, p_{3}\right)$. At least one of $p_{1}, p_{2}$ or $p_{3}$ is not contained by $\Gamma$, otherwise we are in case A1. For any of these three points not contained in $\Gamma$, the direction of the opposite edge is optimal and tight.

Claim 4: If A3 $(\Gamma)$ then $S(\Gamma)=T(\Gamma)=S\left(\Gamma^{\prime}\right)$. If $\Gamma^{\prime}$ is not a thin triangle then it follows from Proposition 11(a) and Lemma 10. If $\Gamma^{\prime}$ is a thin triangle then it follows from Proposition 11(b).

The multiple adjoints $\Gamma^{i}$ for $i \in \mathbf{Z}_{>0}$ are defined in Definition 4. We define An(Z) for $n=0,1,2,3,4$ to be as in Definition 45, but with $\Gamma$ replaced by $Z$ and $\Gamma^{\prime}$ by $Z^{\prime}$. Let

$$
\alpha: \mathcal{V} \rightarrow \mathbf{Z}_{\geq 0}, \quad \Gamma \mapsto \min _{i \geq 0}\left\{i \geq 0 \text { and }\left(\mathrm{A} 2\left(\Gamma^{i+1}\right) \text { or } \mathrm{A} 3\left(\Gamma^{i+1}\right)\right)\right\}
$$

where $\mathcal{V}$ is the set of all convex lattice polygons.

Claim 5: If $\mathrm{A} 4(\Gamma)$ then $T\left(\Gamma^{\prime}\right) \neq \emptyset$ and $S(\Gamma)=S\left(\Gamma^{\prime}\right)$. Induction claim: $C[i]$ : If $\alpha(\Gamma)=i$ and $\mathrm{A} 4(\Gamma)$ then $T\left(\Gamma^{\prime}\right) \neq \emptyset$ and $S(\Gamma)=S\left(\Gamma^{\prime}\right)$, for all $\Gamma$. Induction basis $C$ [0]: From Claims 3, 4 it follows that $S\left(\Gamma^{\prime}\right)=T\left(\Gamma^{\prime}\right)$. From Claim 2 is follows that $C[0]$ holds for both cases. Induction step $(C[i-1] \Rightarrow C[i]$ for $i>0)$ : We are in case A4 $\left(\Gamma^{\prime}\right)$. From the induction hypothesis $C[i-1]$ it follows that $T\left(\Gamma^{2}\right) \neq \emptyset$. From Claim 1 it follows that $S\left(\Gamma^{\prime}\right)=T\left(\Gamma^{\prime}\right) \neq \emptyset$. From Claim 2 it follows that $S(\Gamma)=$ $S\left(\Gamma^{\prime}\right)$.

Remark 14 (Maximal number of optimal viewangles) From Proposition 11 and Fig. 4(16) it follows that $\# S(\Gamma) \leq 4$ for all convex lattice polygons $\Gamma \subset \mathbf{R}^{2}$. Recently [2] proved a generalization of this result to higher dimension. They give an upper bound of $\left(3^{d}-1\right) / 2$ for the number of optimal viewangles for the more general $d$-dimensional convex bodies in $\mathbf{R}^{d}$. Moreover they show that the upper bound is only reached by the regular cross polytopes. 


\section{Families}

Definition 15 (Family of subsets) A family of subsets $\tilde{F}$ of $\tilde{X}$ is defined as the map

$$
\chi: \tilde{I} \rightarrow \mathcal{P}(\tilde{X}), \quad i \mapsto \tilde{F}_{i},
$$

where $\tilde{X}$ is a set, $\tilde{I}$ is a set, $\tilde{U}$ is a subset of $\tilde{I} \times \tilde{X}$ and $\tilde{F}_{i}:=\{x \in \tilde{X} \mid(i, x) \in \tilde{U}\}$ for $i \in \tilde{I}$. We defined $\tilde{F}$ to give some intuition for Definition 16 .

Definition 16 (Family) A family $F$ of $X$ is defined as $\left(F_{i}\right)_{i \in I}$ where $X$ is a projective surface over the field $\mathbf{C}$ of complex numbers, $I$ is a nonsingular curve, $U$ is an irreducible, codimension 1, algebraic subset of $I \times X$ and $F_{i}=\pi_{2 *} \circ \pi_{1}^{-1}(\{i\})$ is an irreducible, codimension 1, algebraic subset of $X$ for generic $i \in I$. The maps $\pi_{1}: U \rightarrow I$ and $\pi_{2}: U \rightarrow X$ denote the first respectively second projection of $U$. We define Fam $X$ to be the set of all families on $X$.

Definition 17 (Degree and geometric genus of a family) Let $F=\left(F_{i}\right)_{i \in I}$ be a family as defined in Definition 16. The degree of a family with respect to a given embedding $X \subset \mathbf{P}^{r}$ is defined as $\operatorname{deg} F:=\operatorname{deg} F_{i}$ for generic $i$. The geometric genus of a family is defined as $p_{g} F:=p_{g} F_{i}$ for generic $i$.

Definition 18 (Attributes of families: fibration and rational) Let $F=\left(F_{i}\right)_{i \in I}$ be a family as defined in Definition 16 . We call $F$ a fibration family if and only if there exists a rational map

$$
f: X \rightarrow-\rightarrow I
$$

such that $F_{i}=f^{-1}(i)^{-}$for all $i \in I$. We call $F$ a rational family if and only if $p_{g} F=0$.

Proposition 19 (Properties of families) Let $F \in$ Fam $X$ be a family.

(a) We see that $\left(F_{i}\right)_{i \in I}$ and $U$ are different representations for the same family $F$.

(b) We have $\operatorname{supp}\left(F_{i}\right)=\{x \in X \mid(i, x) \in U\}$.

(c) If $F$ is a fibration family then $\pi_{2}$ is birational and $f=\pi_{1} \circ \pi_{2}^{-1}$ is a fibration map.

(d) If $X$ is nonsingular then $F_{i}$ is a Cartier divisor for all $i \in I$.

(e) If $X$ is nonsingular then $U \subset X \times I$ is a Cartier divisor.

(f) If $X$ is nonsingular then $\operatorname{deg} F=\operatorname{deg} F_{i}$ for all $i \in I$ and $p_{g} F=\max \left\{p_{g}\left(F_{i}\right) \mid\right.$ $i \in I\}$.

Proof We have that (a) until (e) are straightforward. See [7] Corollary III.9.10 for the proof of (f).

Proposition 20 (Properties of rational families) Let $X$ be nonsingular. Let $K$ be the canonical divisor class of $X$. Let $F \in$ Fam $X$ be a family.

$$
\text { If } p_{g}(F)=0 \text { then } F K \leq-2 \text {. }
$$


Proof Let $U \subset I \times X$ be the Cartier divisor defining $F$. Let $g: \tilde{U} \rightarrow U$ be the resolution of singularities of $U$ (see [7] for resolution of singularities). Let $\rho_{1}:=$ $\pi_{1} \circ g: \tilde{U} \rightarrow I$ and $\rho_{2}:=\pi_{2} \circ g: \tilde{U} \rightarrow X$. Let $G=\left(G_{i}\right)_{i \in I} \in$ Fam $\tilde{U}$ where $G_{i}=g^{*} \pi_{1}^{-1}(\{i\})$.

Claim 1: We have $G^{2}=0$. We have $\rho_{1}\left(G_{i}\right) \neq \rho_{1}\left(G_{j}\right)$ for all $i, j \in I$ such that $i \neq j$. From $\rho_{1}$ being a morphism it follows that $G_{i} \cap G_{j}=\emptyset$ for all $i, j \in I$ such that $i \neq j$.

Claim 2: If $p_{g}(F)=0$ then $p_{a}(G)=0$. From $F_{i}=\pi_{2} \circ \pi_{1}^{-1}(\{i\})$ it follows that $\pi_{2}: g\left(G_{i}\right) \stackrel{\cong}{\rightrightarrows} F_{i}$. It follows that $G_{i}$ and $F_{i}$ are birational for all $i$ and thus $p_{g}(G)=0$. From Sard's theorem it follows that the generic fiber $G_{i}$ of the regular map $\rho_{1}$ is nonsingular. It follows that $p_{a}(G)=p_{g}(G)=0$.

Let $R=K_{\tilde{U}}-\rho_{2}{ }^{*} K_{X}$ be the relative canonical divisor.

Claim 3: We have $G R \geq 0$. Since we can pull back differential forms along a morphism it follows that $0 \rightarrow \rho_{2}^{*} \omega_{X} \rightarrow \omega_{\tilde{U}}$. From the tensor product with an invertible sheaf being exact it follows that $0 \rightarrow O_{X} \rightarrow \omega_{\tilde{U}} \otimes\left(\rho_{2}^{*} \omega_{X}\right)^{-1}$ is exact. From the global section functor being left exact it follows that $\omega_{\tilde{U}} \otimes\left(\rho_{2}^{*} \omega_{X}\right)^{-1}=O_{\tilde{U}}(R)$ is effective. From $G$ having no fixed components and being movable it follows that $G$ is nef and thus $G R \geq 0$.

Let (AF) denote the Adjunction Formula: $p_{a}(C)=\frac{1}{2}\left(C^{2}+C K\right)+1$ for all irreducible curves $C \subset X$ (see [7]).

Claim 4: If $p_{g}(F)=0$ then $F K \leq-2$. From (AF) and Claim 2 it follows that $G K_{\tilde{U}}=2 p_{a}(G)-G^{2}-2=-2$. We have $F K_{X}=\rho_{2 *} G K_{X}=G \rho_{2}{ }^{*} K_{X}=G K_{\tilde{U}}-$ $G R \leq-2$.

Example 21 (Fibration family) Let $X=\mathbf{P}^{2}$. Let $I=\mathbf{P}^{1}$. Let $U=\left\{\left(i_{0}: i_{1}\right) \times\left(x_{0}: x_{1}\right.\right.$ : $\left.\left.x_{2}\right) \mid x_{0} i_{1}=x_{1} i_{0}\right\}$.

The corresponding family $F$ is the family of lines through a point.

It is a fibration family with fibration map $f: X \rightarrow-\rightarrow,\left(x_{0}: x_{1}: x_{2}\right) \longmapsto\left(x_{0}: x_{1}\right)$.

Example 22 (Non-fibration family) Let $X=\mathbf{P}^{2}$. Let $I: i_{0}^{2}+i_{1}^{2}-i_{2}^{2}=0 \subset \mathbf{P}^{2}$. Let $U=\left\{\left(i_{0}: i_{1}: i_{2}\right) \times\left(x_{0}: x_{1}: x_{2}\right) \mid i_{0} x_{0}+i_{1} x_{1}-i_{2} x_{2}=0\right\}$.

The corresponding family $F=\left(F_{i}\right)_{i \in I}$ is the family of tangents to a circle in a plane.

The family $F$ is not a fibration family.

The intersection of two lines is varying with the pair of lines. In other words, generic points in $X$ are reached by 2 family members $F_{i}$.

Definition 23 (Operations on families) Let $F \in \operatorname{Fam} X$ as in Definition 16. Let $f$ : $X \rightarrow Y$ be a birational morphism between projective surfaces. The pushforward of families is defined as

$$
f_{\circledast}: \operatorname{Fam} X \rightarrow \operatorname{Fam} Y, \quad U \mapsto \hat{f}(U) .
$$

The pullback of families is defined as

$$
f^{\circledast}: \operatorname{Fam} Y \rightarrow \operatorname{Fam} X, \quad V \mapsto \overline{\hat{f}^{-1}(V-B)},
$$


where $\hat{f}: I \times X \rightarrow I \times Y,(i, x) \mapsto(i, f(x))$ and $B \subset I \times Y$ is the locus where $\hat{f}^{-1}$ is not defined. If $X$ is nonsingular then the intersection products are defined as $\cdot: \operatorname{Div} X \times \operatorname{Fam} X \rightarrow \mathbf{Z},(D, F) \mapsto D F_{i}$ for any $i \in I$ and $\cdot: \operatorname{Fam} X \times \operatorname{Fam} X \rightarrow \mathbf{Z}$, $\left(F, F^{\prime}\right) \mapsto F_{j} F_{i}^{\prime}$ for any $i \in I$ and $j \in I^{\prime}$. The following proposition shows that the intersection products are well defined.

Proposition 24 (Properties of operations on families) Let $h: X \rightarrow Y$ be a birational morphism between surfaces.

(a) The maps $h_{\circledast}$ and $h^{\circledast}$ are well defined.

(b) We have $h^{\circledast} \circ h_{\circledast}=\operatorname{id}_{\text {Fam } X}$ and $h_{\circledast} \circ h^{\circledast}=\operatorname{id}_{\text {Fam } Y}$.

(c) If $X$ and $Y$ are nonsingular then $f_{\circledast}:$ Fam $X \rightarrow$ Fam $Y,\left(F_{i}\right)_{i \in I} \mapsto\left(f_{*} F_{i}\right)_{i \in I}$, and $f^{\circledast}:$ Fam $Y \rightarrow \operatorname{Fam} X,\left(F_{i}\right)_{i \in I} \mapsto \overline{\left(\left(f^{*} F_{i}\right)_{i \in I}-\left(\bigcap_{i \in I} F_{i}\right)\right)}$ where $f^{*}$ and $f_{*}$ are defined by the pullback and pushforward of divisors.

(d) If $X$ is nonsingular then $D F_{i}=D F_{j}$ for all $D \in \operatorname{Div} X$ and $i, j \in I$ and thus the intersection products are well defined.

Proof We see that (a), (b) and (c) are a straightforward consequence of the definitions. See [7] for the proof of (d) (family members $F_{i}$ are algebraic equivalent and algebraic equivalence implies numerical equivalence).

\section{Minimal degree families on toric surfaces}

Remark 25 (Toric varieties) For the definition of toric varieties we follow [1, 3] and [4]. If $\Gamma$ is a lattice polygon with lattice points $\left\{\left(a_{0}, b_{0}\right), \ldots,\left(a_{r}, b_{r}\right)\right\}$, then the toric surface defined by $\Gamma$ is the projective closure of the image of the map

$$
p: \mathbf{C}^{* 2} \rightarrow \mathbf{P}^{r},(s, t) \mapsto\left(s^{a_{0}} t^{b_{0}}: \cdots: s^{a_{r}} t^{b_{r}}\right)
$$

(see [1, Sect. 12]).

Definition 26 (Attributes of families: toric family) Let $F$ in Fam $X$ be a family as defined in Definition 16. We call $F$ a toric family if and only if $F$ is a fibration family and after resolution of basepoints the fibration map is a toric morphism. Note that $X$ and $I$ have to be toric and in particular $I=\mathbf{P}^{1}$ (see [3] for the definition of toric morphism). The fibration map induces a toric morphism between the dense tori in $X$ and $I$ (see Example 30 below).

Definition 27 (Minimal toric degree and optimal toric family) Let $X$ be a complex embedded toric surface. The minimal toric degree $v(X)$ of $X$ is the smallest possible degree of a toric family on $X$ (see Definition 18). The set of optimal toric families on $X$ is defined as

$$
S(X)=\{F \in \operatorname{Fam} X \mid F \text { is a toric family and } \operatorname{deg} F=v(X)\} .
$$


Fig. 6 Example of toric families and viewangles relation

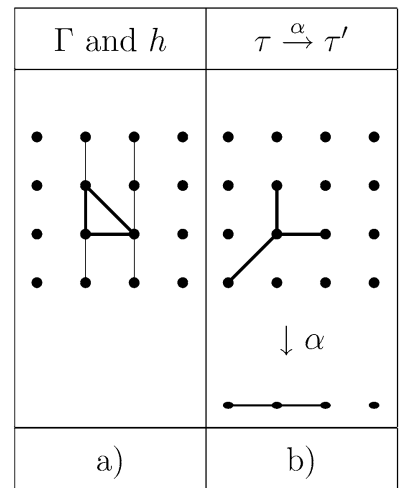

Definition 28 (Toric family problem on toric surfaces) Given a complex embedded toric surface $X$ find the minimal toric degree $v(X)$ and the set of optimal toric families $S(X)$.

Definition 29 (Viewangles and toric families relation) Let $\Gamma$ be a lattice polygon with lattice $\Lambda$. Let $X$ be the toric surface defined by $\Gamma$ (see Remark 25). Let $V$ be the set of primitive viewangles in $\Lambda^{*}-\{0\}$. Let $T$ be the set of toric families on $X$. The viewangles and toric families relation is a function:

$$
\theta_{\Gamma}: V \rightarrow T
$$

where any primitive viewangle $h \in V$ is sent to a toric family in $\theta_{\Gamma}(h) \in T$ in the following way: Let $\Sigma$ with lattice $\Lambda^{*}$ be the normal fan of $\Gamma$ (see [1, Sect. 12]). Let $\Sigma^{\prime}$ be the fan of $\mathbf{P}^{1}$ (the unique projective toric curve) with lattice points in $\Lambda^{*} / h$. Let $\tau$ and $\tau^{\prime}$ be the cones in $\Sigma$ respectively $\Sigma^{\prime}$ corresponding to the dense torus embeddings (thus the cones are points). The canonical linear map $\Lambda^{*} \rightarrow \Lambda^{*} / h$ induces map of fans $\alpha: \tau \rightarrow \tau^{\prime}$ (see [3, Sect. V.4] for map of fans). Let $\beta: X_{\tau} \rightarrow X_{\tau^{\prime}}$ be the toric morphism corresponding to the map of fans $\alpha$ (see [3, Sect. VI.6]). Let $f: X_{\Sigma} \rightarrow X_{\Sigma^{\prime}}$ be the rational map corresponding to the closure of $\beta$. The toric family $\theta_{\Gamma}(h)$ is defined by the fibers of $f$.

Example 30 (viewangles and toric families relation) Let $\theta_{\Gamma}: V \rightarrow T$ be the viewangles and toric families relation. We use the same notation as in Definition 29.

We assume that $\Gamma$ with lattice $\Lambda$ is the standard triangle in Fig. 6(a). The vertical lines represent the viewangle $h=(m, n)=(0,-1)$ in $V$.

The triangle polytope in Fig. 6(a) corresponds to the closure of the image of

$$
p: \mathbf{C}^{* 2} \rightarrow \mathbf{P}^{2}, \quad(s, t) \mapsto(s: t: 1)
$$

which is $\mathbf{P}^{2}$.

In Fig. 6(b) is the normal fan $\Sigma$ of the triangle polygon with lattice $\Lambda^{*}$. Downstairs is the fan of $\mathbf{P}^{1}$ which is the unique projective toric curve, with lattice $\Lambda^{*} / h$.

The canonical linear map $\Lambda^{*} \rightarrow \Lambda^{*} / h$ is defined by the matrix $[n m]=[-10]$, which is the vertical projection. 
It induces a map of fans $\beta: \tau \rightarrow \tau^{\prime}$ on the dense torus embeddings (see Fig. 6(b)). The map $\beta$ defines a semigroup homomorphism:

$$
\beta^{*}:\left[u, u^{-1}\right] \rightarrow\left[s, t, s^{-1}, t^{-1}\right], \quad u \mapsto s^{-n} t^{m}
$$

We have that $\beta^{*}$ defines the following rational map between the toric varieties:

$$
f^{\prime}: \mathbf{C}^{* 2} \rightarrow \mathbf{C}^{*}, \quad(s, t) \mapsto\left(s^{-n} t^{m}\right) .
$$

The closure of $f^{\prime}$ defines the map

$$
f: \mathbf{P}^{2}-\rightarrow \mathbf{P}^{1}, \quad\left(x_{0}: x_{1}: x_{2}\right) \mapsto\left(x_{0}: x_{2}\right)=\left(x_{0}^{-n} x_{1}^{m} x_{2}^{n-m}: 1\right)
$$

which is not defined at $(0: 1: 0)$.

The corresponding toric family $\theta_{\Gamma}(h)$ is the family of lines through the point $(0$ : $1: 0)$.

This family has degree 1 and $h$ is an optimal viewangle of width 1 (see Fig. 4(17)). This is no coincidence as we shall see in Proposition 31.

Proposition 31 (Viewangles and toric families relation) Let $\theta_{\Gamma}: V \rightarrow T$ be the viewangles and toric families relation.

(a) We have that $\theta_{\Gamma}$ is a bijection and a viewangle of width $n$ is sent to a toric family of degree $n$.

Proof We use the same notation as in Definition 29. Let $\left\{\left(a_{0}, b_{0}\right), \ldots,\left(a_{r}, b_{r}\right)\right\}$ be the set of lattice points of $\Gamma$. Let $p: \mathbf{C}^{* 2} \rightarrow \mathbf{P}^{r},(s, t) \mapsto\left(s^{a_{0}} t^{b_{0}}: \ldots: s^{a_{r}} t^{b_{r}}\right)$ (see Remark 25). Let $h=(m, n)$ in $V$ be a primitive viewangle (id est $\operatorname{gcd}(m, n)=1)$. Let $F=\theta_{\Gamma}(h)$. Let

$$
f: X \rightarrow \mathbf{P}^{1}, \quad\left(x_{0}: \cdots: x_{r}\right) \mapsto\left(x_{0}{ }^{e_{0}} \ldots x_{r}{ }^{e_{r}}: 1\right)
$$

such that $\sum e_{i}=0, \sum a_{i} e_{i}=-n$ and $\sum b_{i} e_{i}=m$ for $i \in\{0, \ldots, r\}$.

Claim 1: We have that $f$ is the fibration map of toric family $F$. In Example 30 the map $f$ is obtained for a special case. To see that this construction holds in general is left to the reader.

Let $q=\left(q_{0}: q_{1}\right) \in \mathbf{P}^{1}$.

Claim 2: The fibers $f(q)^{-1}$ are $F_{q}:=\overline{\left\{x \in X \mid x_{0} e_{0} \ldots x_{n} e_{n}=\frac{q_{0}}{q_{1}}\right\}}$. This claim is a direct consequence of the definitions.

Claim 3: We have that $p^{-1}\left(F_{q}\right): s^{-n} t^{m}-\frac{q_{0}}{q_{1}}=0$ and this curve is irreducible if and only if $\operatorname{gcd}(m, n)=1$. If $\alpha, \beta$ are coprime and $z \in \mathbf{Z}_{>1}$ then $s^{z \alpha} t^{z \beta}-1=$ $\left(s^{\alpha} t^{\beta}-1\right)\left(\sum_{i=0}^{i=z} s^{i \alpha} t^{i \beta}\right)$.

Let $k, l \in \mathbf{C}^{*}$ be such that $\frac{q_{0}}{q_{1}}=\frac{l^{m}}{k^{n}}$. Let $h_{q}: \mathbf{C}^{*} \rightarrow \mathbf{C}^{* 2}, u \mapsto\left(k \cdot u^{m}, l \cdot u^{n}\right)$.

Claim 4: The map $h_{q}$ is a birational parametrization of $p^{-1}\left(F_{q}\right)$. This claim is a direct consequence of the definitions.

Let $g_{m n}(q): \mathbf{C}^{*} \rightarrow F_{q}, u \mapsto\left(k^{a_{0}} l^{b_{0}} \cdot u^{a_{0} m+b_{0} n}: \cdots: k^{a_{r}} l^{b_{r}} \cdot u^{a_{r} m+b_{r} n}\right)$. 
Claim 5: The map $g_{m n}(q)$ is a birational parametrization of $F_{q}$ for all generic $q \in \mathbf{P}^{1}$. We have $g_{m n}(q)=p \circ h_{q}$ for all $q \in \mathbf{P}^{1}$. We have $f \circ g_{m n}(u)=$ $k^{\sum a_{i} e_{i}} l^{\sum b_{i} e_{i}} u^{\sum a_{i} e_{i} m+\sum b_{i} e_{i} n}$. It follows that $\sum a_{i} e_{i}=-n$ and $\sum b_{i} e_{i}=m$.

Claim 6: Changing $k, l$ in $g_{m n}(q)$ such that $\frac{q_{0}}{q_{1}}=\frac{l^{m}}{k^{n}}$ gives rise to a reparameterization of $F_{q}$. This is direct consequence of the definition of $h_{q}$ and that $g_{m n}(q)=p \circ h_{q}$ for all $q \in \mathbf{P}^{1}$.

Claim 7: We have $\operatorname{deg} F=\max _{i}\left(a_{i} m+b_{i} n\right)-\min _{i}\left(a_{i} m+b_{i} n\right)$. From Claim 5 it follows that $\operatorname{deg}(F)$ equals the cardinality of $g_{m n}(q) \cap H$ for any $q$ and generic hyperplane section $H$.

Claim 8: We have that (a). The linear system of equations $\sum a_{i} e_{i}=-n, \sum b_{i} e_{i}=$ $m$ and $\sum e_{i}=0$ has solutions in $\vec{e}$. From Claim 6 it follows that $F$ corresponding to $g_{m^{\prime} n^{\prime}}$ depends uniquely on $a_{i}, b_{i}, m^{\prime}$ and $n^{\prime}$. It follows that $\theta_{\Gamma}(h)$ defines uniquely a family $F$. From Claim 7 it follows that a viewangle of width $n$ is sent to a toric family of degree $n$.

Example 32 (Toric family problem) Let $X$ be a complex embedded toric surface. Let $p: \mathbf{C}^{* 2} \rightarrow X \subset \mathbf{P}^{6}, \quad(s, t) \mapsto\left(s^{0} t^{1}: s^{0} t^{2}: s^{1} t^{0}: s^{1} t^{1}: s^{1} t^{2}: s^{2} t^{0}: s^{2} t^{1}\right)$ a birational monomial parameterization.

For $m=1$ and $n=-1$ we find

$$
f: X \rightarrow \mathbf{P}^{1}, \quad\left(x_{0}: \cdots: x_{6}\right) \mapsto\left(x_{1}^{2} x_{2}: x_{0}^{3}\right)
$$

and $\operatorname{deg}\left(F_{q}\right)=4$ for all $q \in \mathbf{P}^{1}$.

For $m=1$ and $n=0$ we find

$$
f: X \rightarrow \mathbf{P}^{1}, \quad\left(x_{0}: \cdots: x_{6}\right) \mapsto\left(x_{1}: x_{0}\right)
$$

and $\operatorname{deg}\left(F_{q}\right)=2$ for all $q \in \mathbf{P}^{1}$. We have that Examples 32 and 8 reflect an equivalent problem instance.

\section{Adjoint chain}

Remark 33 (References) We claim no new results in this section. For the notion of nef, movable, canonical class and exceptional curve we refer to [7] and [9]. The adjoint chain is a reformulation and adapted version of $(D+K)$-minimalization as described in [8] and can also be found in [10].

Definition 34 (Minimally polarized rational surface (mprs)) A minimally polarized rational surface (mprs) is defined as a pair $(X, D)$ where $X$ is a nonsingular rational surface over $\mathbf{C}, D$ is a nef and movable divisor on $X$ and there does not exists a -1-curve $C$ such that $D C=0$.

Definition 35 (Minimal mprs) Let $(X, D)$ be a mprs. Let $K$ denote the canonical divisor class on $X$. We call $(X, D)$ a minimal mprs if and only if $\operatorname{dim}|D+K| \leq 0$ or $D^{2}=0$. 
Definition 36 (Adjoint relation) Let $(X, D)$ be a mprs which is not minimal. An adjoint relation is a relation $(X, D) \stackrel{\mu}{\rightarrow}\left(X^{\prime}, D^{\prime}\right)$ where $(X, D)$ is a mprs which is not minimal, $\left(X^{\prime}, D^{\prime}\right)$ is a mprs, $X \stackrel{\mu}{\rightarrow} X^{\prime}$ is a birational morphism which blows down all -1-curves $C$ such that $(D+K) C=0$ and $D^{\prime}=\mu_{*}(D+K)$.

Definition 37 (Adjoint chain) An adjoint chain of $(X, D)$ is a chain of adjoint relations until a minimal mprs is obtained:

$$
(X, D)=\left(X_{0}, D_{0}\right) \stackrel{\mu_{0}}{\rightarrow}\left(X_{1}, D_{1}\right) \stackrel{\mu_{1}}{\rightarrow} \ldots
$$

Proposition 38 (Properties of adjoint chain)

(a) The adjoint chains of a mprs are finite and have the same length.

(b) If $(X, D) \stackrel{\mu}{\rightarrow}\left(X^{\prime}, D^{\prime}\right)$ is an adjoint relation then $\mu^{*} D^{\prime}=D+K$.

Proof The proofs can be found in [10].

\section{Minimal degree families on polarized rational surfaces}

Definition 39 (Optimal and tight families and minimal degree) Let $(X, D)$ be a mprs. Let $K$ be the canonical divisor class on $X$. Let $F \in \operatorname{Fam}(X)$. The degree of $F$ with respect to $(X, D)$ is given by $D F$. We call $F$ a tight family if and only if $F K=-2$. The minimal rational degree with respect to $(X, D)$ is defined as

$$
v(X, D)=\min \left\{D F \mid F \in \operatorname{Fam} X \text { and } p_{g}(F)=0\right\} .
$$

The minimum exists since $D$ is nef by definition. We call $F$ an optimal family if and only if $F$ is a rational family and $D F=v(X, D)$. The set of all optimal families on $(X, D)$ is denoted by $S(X, D)$.

Example 40 (Optimal families of the projective plane) Let $F$ be the family of lines through a point (see Example 21). Let $L$ be the divisor class of lines on $\mathbf{P}^{2}$.

We have that $\left(\mathbf{P}^{2}, L\right)$ is a mprs.

We have $F \in S\left(\mathbf{P}^{2}, L\right)$ and $v\left(\mathbf{P}^{2}, L\right)=F L=1$.

Definition 41 (Rational family problem on mprs) Given a mprs $(X, D)$ find the minimal degree $v(X, D)$ and all optimal families $S(X, D)$.

Lemma 42 (Lowerbound) Let $(X, D) \stackrel{\mu}{\rightarrow}\left(X^{\prime}, D^{\prime}\right)$ be an adjoint relation. Let $F \in$ Fam $X$ be a rational family.

We have $F D \geq \mu_{\circledast} F D^{\prime}+2$, and equality holds if and only if $F$ is tight.

Proof From Proposition 38(b) and Proposition 20 it follows that $\mu_{\circledast} F D^{\prime}=F \mu^{*} D^{\prime}=$ $F D+F K \leq F D-2$. 
Lemma 43 (Tight) Let $\mu: X \rightarrow X^{\prime}$ a birational morphism between nonsingular complex projective surfaces. Let $F^{\prime} \in \mathrm{Fam} X^{\prime}$ be tight.

(a) We have that $\mu^{\circledast} F^{\prime} \in \operatorname{Fam} X$ is tight.

(b) We have $\mu^{\circledast} F^{\prime}=\left(\mu^{*} F_{i}^{\prime}\right)_{i \in I}$.

Proof

Claim: We assume without loss of generality that $\mu=\pi$ where $\pi$ blows down one exceptional curve $E$.

Claim: We have that (a) and (b). We have $\mu^{\circledast} F^{\prime} K=F^{\prime} \mu_{*} K=F^{\prime} K^{\prime}=-2$. We have $\mu^{\circledast} F^{\prime} K=(F+m E) K=F K-m=-2$ where $m \geq 0$. From Proposition 20 it follows that $m=0$ and $F K=-2$.

Proposition 44 (Classification optimal fibration families on minimal mprs)

All the optimal fibration families on minimal mprs $(X, D)$ are classified in the following table:

\begin{tabular}{lllllll}
\hline$D$ & $D^{2}$ & $X \cong \mathbf{P}^{2}$ & Optimal families $F$ & $D F$ & Tight & Type \\
\hline$D=n P$ & 0 & No & $F=P$ & 0 & Yes & Ruled \\
$2 D+K=n P$ & $n+2$ & No & $F=P$ & 1 & Yes & Linear fibration \\
$2 D+K=0$ & 2 & No & 1 or 2 families of lines & 1 & Yes & Linear fibration \\
$D=L$ & 1 & Yes & $F \subset L$ & 1 & No & Plane \\
$D=2 L$ & 2 & Yes & $F \subset L$ & 2 & No & Plane \\
$D+K=0$ & 9 & Yes & $F \subset L$ & 3 & No & Plane \\
$D+K=0$ & $1,2, \ldots, 8$ & No & See $[11]$ & 2 & Yes & Conic fibration \\
$D+c K=0$ & 0 & No & Infinitely many & $2 c$ & Yes & \\
\hline
\end{tabular}

where $p_{g}(L)=0, \operatorname{dim}|L|=2$ and $L^{2}=1$ ( $L$ stands for lines); and $p_{g}(P)=0$, $\operatorname{dim}|P|=1$ and $P^{2}=0$ and $c \in \mathbf{Z}_{>0}$. In particular we see that there is always an optimal family of fibration type.

Proof The first 3 columns are known from [8]. The third row denotes families of lines of a quadric surface in $\mathbf{P}^{3}$. The rows 4 to 7 are known from [11, pp. 81-85]). The cases $D^{2}=1,2$ in row 7 are not covered in [11], but are straightforward generalizations. The last row is the Halphen pencil and can be found in [6] and Exercise V.4.15.e in [7]. This pair can never arise as a last link in an adjoint chain where the mprs $\left(X_{0}, D_{0}\right)$ satisfies $D_{0}^{2}>0$. Let (AF) denote the Adjunction Formula: $p_{a}(C)=\frac{1}{2}\left(C^{2}+C K\right)+1$ for all irreducible curves $C \subset X$ (see [7]). Let $F=\left(F_{i}\right)_{i \in I}$ in Fam $X$ be any family such that $F P=0$.

Claim 1: We have $F=P$. From $F P=0$ and $F, P$ being movable it follows that there exist curves $C \in|P|$ and $F_{j} \in F$ through some generic point $x \in X$. From $C F_{j}=0$ and $x \in C \cap F_{j}$ it follows that $C=F_{j}$ and thus $F=P$.

Claim 2: If $D=n P$ then $P$ is the unique optimal tight fibration family. From (AF) it follows that $p_{a}(P)=\frac{1}{2}(0+P K)+1=0$, and thus $P K=-2$. From $D$ being nef 
and $D P=0$ it follows that $P$ is an optimal family. The fibration map is given by $\varphi_{|D|}$. From Claim 1 it follows that $P$ is the unique optimal family.

Claim 3: If $2 D+K=n P$ then $P$ is the unique optimal tight fibration family. We have $F(2 D+K) \geq 0$ for all $F \in S(X, D)$. From Proposition 20 it follows that $2 F D=F(2 D+K)-F K \geq 0+2$ and thus $F D \geq 1$. If $F=P$ then $F D=1$. If $F D=1$ then $2 F D=F(2 D+K)-F K=2$ and thus $F P=0$. From Claim 1 it follows that $P$ is the unique optimal family.

Definition 45 (Case distinction) Let $(X, D) \stackrel{\mu}{\rightarrow}\left(X^{\prime}, D^{\prime}\right)$ be an adjoint relation. We distinguish the following cases where $(X, D)$ is not minimal except at B0:

\begin{tabular}{lll}
\hline & $(X, D)$ & $\left(X^{\prime}, D^{\prime}\right)$ \\
\hline B0 & Minimal mprs & - \\
B1 & $X \cong \mathbf{P}^{2}$ & $X^{\prime} \cong \mathbf{P}^{2}$ \\
B2 & $X \nsucceq \mathbf{P}^{2}$ & $X^{\prime} \cong \mathbf{P}^{2}$ \\
B3 & $X \varsubsetneqq \mathbf{P}^{2}$ & Minimal mprs and $X^{\prime} \nsucceq \mathbf{P}^{2}$ \\
B4 & $X ¥ \mathbf{P}^{2}$ & Not minimal mprs and $X^{\prime} \nsucceq \mathbf{P}^{2}$ \\
\hline
\end{tabular}

Theorem 46 (Optimal families and minimal degree) Let $(X, D) \stackrel{\mu}{\rightarrow}\left(X^{\prime}, D^{\prime}\right)$ be an adjoint relation. Let $\mathrm{B} 0$ until $\mathrm{B} 4$ denote the cases as in Definition 45 . Let $L$ be the divisor class of lines on $X$, if $X \cong \mathbf{P}^{2}$. Let $L_{p}^{\prime}$ be the family of lines through the point $p$ for any $p \in X^{\prime}$, if $X^{\prime} \cong \boldsymbol{P}^{2}$. Let $B$ be the set of indeterminacy points of $\mu^{-1}$.

(a) If $\mathrm{B} 0$ then $S(\Gamma)$ and $v(\Gamma)$ are given by Proposition 44.

(b) If B1 then $S(X, D)=\{F \mid F \subset L\}$ and $v(X, D)=v\left(X^{\prime}, D^{\prime}\right)+3$.

(c) If $\mathrm{B} 2$ then $S(X, D)=\left\{\mu^{\circledast} L_{p}^{\prime} \mid p \in B\right\}$ and $v(X, D)=v\left(X^{\prime}, D^{\prime}\right)+2$.

(d) If $\mathrm{B} 3$ or $\mathrm{B} 4$ then $S(X, D)=\left\{\mu^{\circledast} F^{\prime} \mid F^{\prime} \in S\left(X^{\prime}, D^{\prime}\right)\right\}$ and $v(X, D)=$ $v\left(X^{\prime}, D^{\prime}\right)+2$.

Proof We have that (a) is a direct consequence of Proposition 44. We have that (b) follows from Claim 1, (c) follows from Claim 5 and (d) follows from Claim 8 and Claim 9, where the claims are given below. Let $L$ and $L^{\prime}$ be the class of lines on respectively $X$ and $X^{\prime}$, if $X \cong \mathbf{P}^{2}$ or $X^{\prime} \cong \mathbf{P}^{2}$.

Claim 1: If B1 then $F \subset L$ and $v(X, D)=v\left(X^{\prime}, D^{\prime}\right)+3$. If $X \cong \mathbf{P}^{2}$ then $F \subset L$ for all $F \in S(X, D)$. From Lemma $43(\mathrm{~b})$ and $K_{\mathbf{P}^{2}}=-3 L$ it follows that $L^{\prime} D^{\prime}=$ $\mu^{*} L^{\prime} \mu^{*} D^{\prime}=L(D+K)=L D-3$.

Let $(\tilde{X}, \tilde{D}) \stackrel{g}{\rightarrow}\left(X^{\prime}, D^{\prime}\right)$ be a relation such that $g: \tilde{X} \rightarrow X^{\prime}$ is the blowup of a point $p \in B$ and $\tilde{D}=g^{*} D^{\prime}$. Let $(X, D) \stackrel{f}{\rightarrow}(\tilde{X}, \tilde{D})$ be a relation such that $\mu=g \circ f$ and $\tilde{D}=f_{*}(D+K)$.

Claim 2: The relation $(X, D) \stackrel{f}{\rightarrow}(\tilde{X}, \tilde{D}) \stackrel{g}{\rightarrow}\left(X^{\prime}, D^{\prime}\right)$ where $\mu=g \circ f$ exists. It follows from [7, Proposition V.5.3 (factorization of birational morphisms)]. 
Let $G_{p}^{\prime}=L_{p}^{\prime}$ and $\tilde{G}_{p}=g^{\circledast} G_{p}^{\prime}=\left(g^{*} G_{p i}^{\prime}\right)_{i \in I}-\tilde{E}$ and $G_{p}=f^{\circledast} \tilde{G}_{p}=\mu^{\circledast} G_{p}^{\prime}$ for $p \in B$ which is blown up by $g$. Let $R(X, D)=\left\{G_{p} \mid p \in B\right\}$. Let $T(X, D)=\{F \mid$ $F \in S(X, D)$ and $F$ is tight $\}$.

Claim 3: If B2 then $R(X, D) \subseteq T(X, D)$. We have $\tilde{G}_{p} \tilde{K}=\left(\left(g^{*} G_{p i}^{\prime}\right)_{i \in I}-\tilde{E}\right) \tilde{K}=$ $G_{p}^{\prime} g_{*} \tilde{K}+1=-2$. From Lemma 43(b) it follows that $\left(f^{*} \tilde{G}_{p i}\right)_{i \in I}=f^{\circledast} \tilde{G}_{p}=G_{p}$. It follows that $G_{p} K=f^{\circledast} \tilde{G}_{p} K=\tilde{G}_{p} f_{*} K=-2$. We have $\tilde{G}_{p} \tilde{D}=\left(g^{*} G_{p i}^{\prime}-\tilde{E}\right) \tilde{D}=$ $g^{*} G_{p i}^{\prime} g^{*} D^{\prime}=G_{p}^{\prime} D^{\prime}$ for all $i \in I$. From Lemma 42 it follows that $G_{p} D=f_{\circledast} G_{p} \tilde{D}+$ $2=\tilde{G}_{p} \tilde{D}+2=G_{p}^{\prime} D^{\prime}+2$. From Claim 1 it follows that $G_{p}^{\prime} D^{\prime}$ is minimal and thus $G_{p} \in T(X, D)$.

Claim 4: If B2 then $R(X, D) \supseteq S(X, D)$. If $F \in S(X, D)$ then $F D \geq \mu_{\circledast} F D^{\prime}+2$ and thus $\mu_{\circledast} F \subset L^{\prime}$. It follows that $F D=\mu^{\circledast} \mu_{\circledast} F D=L^{\prime} D^{\prime}+2$ where $\left(\mu^{*} \mu_{*} F_{i}\right)_{i \in I} K=-3$. From Lemma 42 it follows that $\left(\mu^{*} \mu_{*} F_{i}\right)_{i \in I} D>L^{\prime} D^{\prime}+2$. It follows that $\mu^{\circledast} \mu_{\circledast} F \neq\left(\mu^{*} \mu_{*} F_{i}\right)_{i \in I}$ and they differ by a fixed component, which can only come from $p \in B$.

Claim 5: If B2 then $S(X, D)=T(X, D)=\left\{\mu^{\circledast} L_{p}^{\prime} \mid p \in B\right\}$. It follows from Claim 3 and Claim 4.

Claim 6: If $T\left(X^{\prime}, D^{\prime}\right) \neq \emptyset$ then $S(X, D)=T(X, D)$. From Lemma 42 it follows that if $F^{\prime} \in T\left(X^{\prime}, D^{\prime}\right)$ then $\mu^{\circledast} F^{\prime} D=v\left(X^{\prime}, D^{\prime}\right)+2$. From Lemma 42 it follows that if $F \in S(X, D)$ then $F D \geq v\left(X^{\prime}, D^{\prime}\right)+2$ and equality holds if and only if $F \in$ $T(X, D)$.

Let $\mu^{\circledast} S(X, D)=\left\{\mu^{\circledast} F \mid F \in S(X, D)\right\}$.

Claim 7: If $S\left(X^{\prime}, D^{\prime}\right)=T\left(X^{\prime}, D^{\prime}\right)$ then $S(X, D)=\mu^{\circledast} S\left(X^{\prime}, D^{\prime}\right)$. From Lemma 43 it follows that if $F^{\prime} \in T\left(X^{\prime}, D^{\prime}\right)$ then $\mu^{\circledast} F^{\prime} D=v\left(X^{\prime}, D^{\prime}\right)+2$. It follows that $T(X, D) \supseteq \mu^{\circledast} T\left(X^{\prime}, D^{\prime}\right)$. If $F \in T(X, D)$ then $F D=\mu_{\circledast} F D+2$ and thus $\mu_{\circledast} F \in S\left(X^{\prime}, D^{\prime}\right)$. It follows that $T(X, D) \subseteq \mu^{\circledast} S\left(X^{\prime}, D^{\prime}\right)$. From Claim 6 and the assumption it follows that $S(X, D)=T(X, D)$ and $S\left(X^{\prime}, D^{\prime}\right)=T\left(X^{\prime}, D^{\prime}\right)$.

Claim 8: If B3 then $S(X, D)=T(X, D)=\mu^{\circledast} S\left(X^{\prime}, D^{\prime}\right)$. It follows from Proposition 44, Claim 5 and Claim 6.

We will use the adjoint chain (see Definition 37) and define $(X, D)$ to be $\left(X_{0}, D_{0}\right)$ and $\left(X^{\prime}, D^{\prime}\right)$ to be $\left(X_{1}, D_{1}\right)$. We define $\mathrm{B} n\left(X_{i}, D_{i}\right)$ for $n=0,1,2,3,4$ to be as in Definition 45 , but with $(X, D)$ replaced by $\left(X_{i}, D_{i}\right)$ and $\left(X^{\prime}, D^{\prime}\right)$ replaced by $\left(X_{i+1}, D_{i+1}\right)$. Let

$\alpha: \mathcal{V} \rightarrow \mathbf{Z}_{\geq 0}, \quad(X, D) \mapsto \min _{i \geq 0}\left\{i \mid i \geq 0\right.$ and $\left(\mathrm{B} 2\left(X_{i+1}, D_{i+1}\right)\right.$ or $\left.\left.\mathrm{B} 3\left(X_{i+1}, D_{i+1}\right)\right)\right\}$

where $\mathcal{V}$ is the set of all mprs's. It follows from Proposition 38 that the length of an adjoint chain of $(X, D)$ is unique, and thus $\alpha$ is well defined.

Claim 9: If $\mathrm{B} 4(X, D)$ then $T\left(X^{\prime}, D^{\prime}\right) \neq \emptyset$ and $S(X, D)=\mu^{\circledast} S\left(X^{\prime}, D^{\prime}\right)$. Induction claim: $C[i]$ : If $\alpha(X, D)=i$ and $\mathrm{B} 4(X, D)$ then $T\left(X^{\prime}, D^{\prime}\right) \neq \emptyset$ and $S(X, D)=$ $\mu^{\circledast} S\left(X^{\prime}, D^{\prime}\right)$, for all $(X, D)$. Induction basis $C[0]$ : From Claims 5,8 it follows that $S\left(X^{\prime}, D^{\prime}\right)=T\left(X^{\prime}, D^{\prime}\right)$. From Claim 7 it follows that $C[0]$ holds for both cases. Induction step $(C[i-1] \Rightarrow C[i]$ for $i>0)$ : We are in case $\mathrm{B} 4\left(X_{1}, D_{1}\right)$. From the induction hypothesis $C[i-1]$ it follows that $T\left(X_{2}, D_{2}\right) \neq \emptyset$. From Claim 6 it follows that $S\left(X^{\prime}, D^{\prime}\right)=T\left(X^{\prime}, D^{\prime}\right) \neq \emptyset$. From Claim 2 it follows that $S(X, D)=\mu^{\circledast} S\left(X^{\prime}, D^{\prime}\right)$. 
Remark 47 (Analogy with finding optimal viewangles on vineyards) The analogy between this section and Sect. 3, is stated in the following table:

\begin{tabular}{lll}
\hline Sect. 3 & Sect. 7 & Description \\
\hline Definition 7 & Definition 41 & Problem description \\
Lemma 9 & Lemma 42 & Lower bound \\
Lemma 10 & Lemma 43 & Properties of tight \\
Proposition 11 & Proposition 44 & Classification minimal vineyards/mprs \\
Definition 12 & Definition 45 & Cases A0-A4/B0-B4 \\
Theorem 13 & Theorem 46 & Determining optimal vineyards/optimal families \\
\hline
\end{tabular}

The proof of the geometric statement in this section was modeled as a blueprint of the proof of the combinatorial statement in Sect. 3 (we thank the anonymous referee for the notion of blueprint). The combinatorial proof served us as a guideline to a deeper understanding of the geometric one. As described in Sect. 5, there is a translation of the vineyard problem to the family problem. Under this correspondence, the adjoint polygon (see Definition 4) translates into the definition of the adjoint relation for minimally polarized toric surfaces: the projective embedding defined by the interior lattice points is the embedding associated to the adjoint linear system $D+K$, where $D$ is the divisor defined by the original lattice polygon (see [4]). So, not only the problem but also the theorem and proof translate to toric surfaces. But the so obtained theorem and proof do not use the toric structure and can be generalized to the case of arbitrary rational surfaces.

\section{Minimal degree families on rational surfaces}

Definition 48 (Optimal families and minimal degree) Let $Y \subset \mathbf{P}^{r}$ be a rational complex surface (possibly singular) for $r \in \mathbf{Z}_{>1}$. Let $F \in \operatorname{Fam}(Y)$. The minimal rational degree with respect to $Y \subset \mathbf{P}^{r}$ is defined as

$$
v(Y)=\min \left\{\operatorname{deg} F \mid F \in \operatorname{Fam} Y \text { and } p_{g}(F)=0\right\} .
$$

We call $F$ an optimal family if and only if $F$ is a rational family and $\operatorname{deg} F=v(Y)$. The set of all optimal families on $Y \subset \mathbf{P}^{r}$ is denoted by $S(Y)$.

Definition 49 (Rational family problem on rational surfaces) Given a rational complex surface $Y \subset \mathbf{P}^{r}$, find the minimal degree $v(Y)$ and all optimal families $S(Y)$.

Proposition 50 (Optimal families on rational surfaces) Let $Y \subset \mathbf{P}^{r}$ be a rational complex surface for $r \in \mathbf{Z}_{>1}$. Let $\varphi_{D}: X \rightarrow Y$ be the minimal resolution of singularities of $Y$. Let $D \in \operatorname{Div} X$ be the pullback of hyperplane sections of $Y$. Let $S(X, D)$ and $v(X, D)$ be defined as in Definition 39. Let $\varphi_{D \circledast} S(X, D)=\left\{\varphi_{D \circledast} F \mid F \in S(X, D)\right\}$.

(a) We have that $(X, D)$ is a mprs.

(b) We have $S(Y)=\varphi_{D \circledast} S(X, D)$ and $v(Y)=v(X, D)$. 
Proof

Claim: We have that (a). It follows from $D$ being the pullback of the hyperplane sections of $Y \subset \mathbf{P}^{r}$ that $D$ is nef and movable. It follows from $\varphi_{D}$ being a minimal resolution that $D E>0$ for all exceptional curves $E \subset X$. It follows from the definitions that $X$ is rational and nonsingular.

Claim: We have that (b). It follows from $\varphi_{D}$ being birational and from $\operatorname{deg} \varphi_{D}(C)$ $=D C$ for all curves $C \subset X$.

\section{Examples of minimal degree families on rational surfaces}

Example 51 (Case B2) Let $F, G \in \mathbf{C}$ be homogeneous where $\operatorname{deg} F=d$ and $\operatorname{deg} G=$ $d-1$ for $d \in \mathbf{Z}_{\geq 4}$ and $F$ and $G$ have no common multiple points.

Let

$$
Y: F(y, z, w)-x G(y, z, w)=0 \subset \mathbf{P}^{3}
$$

be a complex projective surface of degree $d$.

We consider the following birational map which parametrizes $Y$ :

$$
f: \mathbf{P}^{2}-\rightarrow Y \subset \mathbf{P}^{3}, \quad(s: t: u) \mapsto(F(s, t, u): s G(s, t, u): t G(s, t, u): u G(s, t, u))
$$

given by polynomials of degree $d$ (also called parametric degree).

We define $g: X \rightarrow \mathbf{P}^{2}$ to be the resolution of the projective plane in the basepoints of $f$. There are $d(d-1)$ basepoints including infinitely near basepoints. We define $D \in \mathrm{Cl} X$ to be associated to the resolution of $f$ which is shown in the following commutative diagram:

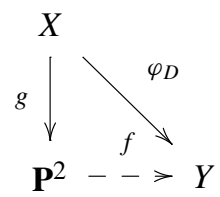

From $\varphi_{D}$ being a morphism it follows that $D$ is nef. Since $|D+K|$ does not have fixed components it follows that $(X, D)$ is a mprs (see Definition 34). Let $E_{1}, \ldots, E_{d(d-1)}$ be the pullbacks of the exceptional curves resulting from blowing up the basepoints of $f$ and $L$ be the pullback of hyperplane sections of $\mathbf{P}^{2}$.

We consider the adjoint relation (see Definition 36)

$$
(X, D) \stackrel{\mu}{\rightarrow}\left(X^{\prime}, D^{\prime}\right)
$$

where $\mathrm{Cl} X=\mathbf{Z}\left\langle L, E_{1}, \ldots, E_{d(d-1)}\right\rangle$ and $\mathrm{Cl} X^{\prime}=\mathbf{Z}\langle L\rangle, K=-3 L+E_{1}+\cdots+$ $E_{d(d-1)}$ and $K^{\prime}=-3 L$ are the canonical divisor classes, $D=d L-E_{1}-\cdots-$ $E_{d(d-1)}$ and $D^{\prime}=(d-3) L$, where $L^{2}=1, E_{i} E_{j}=-\delta_{i j}$ and $L E_{i}=0$ for all $i, j \in$ $\{1, \ldots, 12\}$. Let us assume that $E_{1}, \ldots, E_{r}$ correspond to the planar (not infinitely near) basepoints for $1 \leq r \leq d(d-1)$. 
Fig. 7 Family members of an optimal family on $\tilde{Y}$ in Example 51
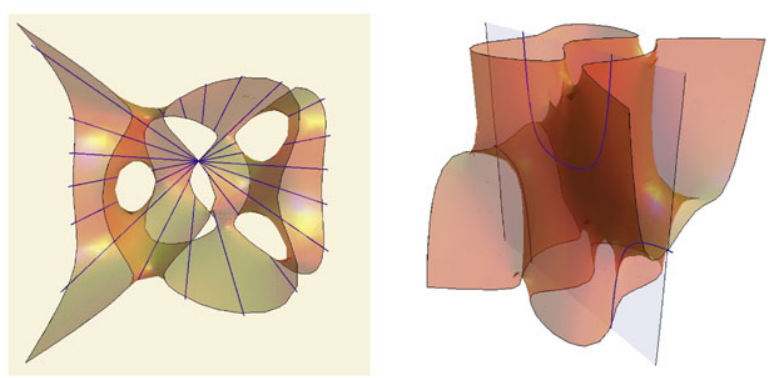

a

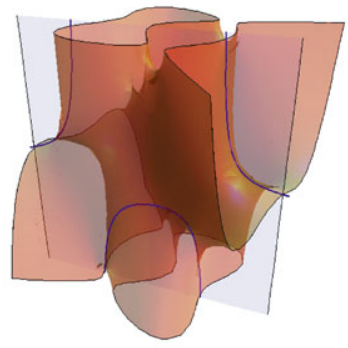

C

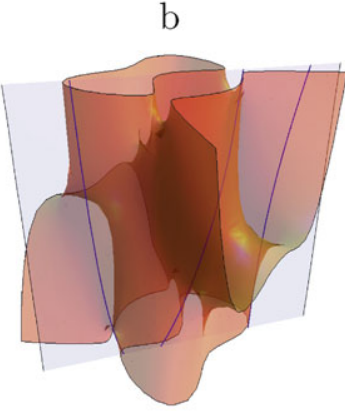

d

From $X^{\prime} \cong \mathbf{P}^{2}$ and Theorem 46(c) case B2 it follows that $S\left(X^{\prime}, D^{\prime}\right)=\{F \subset L\}$ and $v\left(X^{\prime}, D^{\prime}\right)=d-3 . S(X, D)=\left\{\left|L-E_{1}\right|,\left|L-E_{2}\right|, \ldots,\left|L-E_{r}\right|\right\}$ and $v(X, D)=$ $d-1$.

For instance let $\tilde{Y}$ be an affine real representation of $Y$ where $F\left(a_{0}, a_{1}, a_{2}\right)=$ $a_{0}\left(a_{0}+a_{2}\right)\left(a_{0}+2 a_{2}\right)\left(a_{0}+3 a_{2}\right)$ and $G\left(a_{0}, a_{1}, a_{2}\right)=a_{1}\left(a_{1}+a_{2}\right)\left(a_{1}+2 a_{2}\right)$. The images in Fig. 7 show family members of $\left|L-E_{1}\right|$ on $\tilde{Y}$. From the top view in Fig. 7(a) it can be seen that the family is projected to lines through a point in the plane. The exceptional curves $E_{1}, \ldots, E_{12}$ are vertical lines. The family $\left|L-E_{1}\right|$ is given by the hyperplane sections through the vertical line corresponding to $E_{1}$ minus the fixed component which is the line itself. In Fig. 7(b-d) are some hyperplane sections shown corresponding to the family members.

Example 52 (Cases B0, B3 and B4) Let $Y_{0}: w^{6} x^{2}+12 w^{7} y+w^{2} x^{5} y-w^{3} x^{3} y^{2}+$ $4 w^{4} x^{3} z+52 w^{5} x y z-2 w x^{4} y^{2} z+4 w^{2} x^{4} z^{2}+26 w^{3} x^{2} y z^{2}-12 w^{4} y^{2} z^{2}+x^{3} y^{3} z^{2}-$ $20 w x^{3} y z^{3}-28 w^{2} x y^{2} z^{3}-24 w^{3} x z^{4}+17 x^{2} y^{2} z^{4}-48 w x^{2} z^{5}-240 w^{2} y z^{5}+88 x y z^{6}+$ $144 z^{8}=0 \subset \mathbf{P}^{3}$ be a complex projective surface of degree 8 .

We consider the following birational map which parametrizes $Y_{0}$ :

$$
\begin{aligned}
& f_{0}: \mathbf{P}^{2} \rightarrow Y_{0} \subset \mathbf{P}^{3}, \quad(s: t: u) \mapsto \\
& \left(-45 s^{15} t^{4}-21 s^{10} t^{9}-15 s^{5} t^{14}+t^{19}+24 s^{18} u-6 s^{13} t^{5} u+20 s^{8} t^{10} u-6 s^{3} t^{15} u\right. \\
& \quad-44 s^{16} t^{2}-78 s^{11} t^{6} u^{2}-60 s^{6} t^{11} u^{2}+6 s t^{16} u^{2}-6 s^{14} t^{2} u^{3}+60 s^{9} t^{7} u^{3} \\
& \quad-30 s^{4} t^{12} u^{3}-93 s^{12} t^{3} u^{4}-90 s^{7} t^{8} u^{4}+15 s^{2} t^{13} u^{4}+60 s^{10} t^{4} u^{5}-60 s^{5} t^{9} u^{5}
\end{aligned}
$$




$$
\begin{aligned}
& -36 s^{13} u^{6}-60 s^{8} t^{5} u^{6}+20 s^{3} t^{10} u^{6}+20 s^{11} t u^{7}-60 s^{6} t^{6} u^{7}-15 s^{9} t^{2} u^{8} \\
& +15 s^{4} t^{7} u^{8}-30 s^{7} t^{3} u^{9}+6 s^{5} t^{4} u^{10}-6 s^{8} u^{11}+s^{6} t u^{12} \\
: & s^{11} t^{8}+6 s^{6} t^{13}+s t^{18}-12 s^{14} t^{4} u-4 s^{9} t^{9} u+4 s^{17} u^{2}+18 s^{12} t^{5} u^{2}+24 s^{7} t^{10} u^{2} \\
& +6 s^{2} t^{15} u^{2}-12 s^{15} t u^{3}-12 s^{10} t^{6} u^{3}+9 s^{13} t^{2} u^{4}+36 s^{8} t^{7} u^{4}+15 s^{3} t^{12} u^{4} \\
& -12 s^{11} t^{3} u^{5}+24 s^{9} t^{4} u^{6}+20 s^{4} t^{9} u^{6}-4 s^{12} u^{7}+6 s^{10} t u^{8}+15 s^{5} t^{6} u^{8} \\
& +6 s^{6} t^{3} u^{10}+s^{7} u^{12} \\
:- & s^{18} t-9 s^{13} t^{6}-10 s^{8} t^{11}+s^{3} t^{16}-s^{16} t^{2} u+10 s^{11} t^{7} u-5 s^{6} t^{12} u-23 s^{14} t^{3} u^{2} \\
& -30 s^{9} t^{8} u^{2}+5 s^{4} t^{13} u^{2}+20 s^{12} t^{4} u^{3}-20 s^{7} t^{9} u^{3}-14 s^{15} u^{4}-30 s^{10} t^{5} u^{4} \\
& +10 s^{5} t^{10} u^{4}+10 s^{13} t u^{5}-30 s^{8} t^{6} u^{5}-10 s^{11} t^{2} u^{6}+10 s^{6} t^{7} u^{6}-20 s^{9} t^{3} u^{7} \\
& +5 s^{7} t^{4} u^{8}-5 s^{10} u^{9}+s^{8} t u^{10} \\
:- & 12 s^{19}-15 s^{14} t^{5}+10 s^{9} t^{10}+s^{4} t^{15}+4 s^{17} t u-20 s^{12} t^{6} u-15 s^{15} t^{2} u^{2} \\
& +30 s^{10} t^{7} u^{2}+5 s^{5} t^{12} u^{2}-40 s^{13} t^{3} u^{3}+30 s^{11} t^{4} u^{4}+10 s^{6} t^{9} u^{4}-20 s^{14} u^{5} \\
& \left.+10 s^{12} t^{6}+10 s^{7} t^{6} u^{6}+5 s^{8} t^{3} u^{8}+s^{9} u^{10}\right)
\end{aligned}
$$

given by polynomials of degree 19 (also called parametric degree).

We define $g_{0}: X_{0} \rightarrow \mathbf{P}^{2}$ to be the resolution of the projective plane in the basepoints of $f_{0}$. There are 10 basepoints with multiplicities 7, 6, 6, 6, 6, 6, 6, 6, 6 and 4 (this example was constructed by first giving the basepoints with multiplicities and computing the implicit equation afterwards). We define $D_{0} \in \mathrm{Cl} X_{0}$ to be associated to the resolution of $f_{0}$ which is shown in the following commutative diagram:

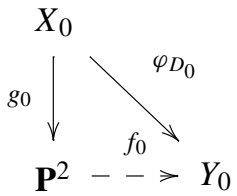

We have that $\left(X_{0}, D_{0}\right)$ is a mprs by the same argument as in Example 51.

We consider the adjoint chain $\left(X_{0}, D_{0}\right) \stackrel{\mu_{0}}{\rightarrow}\left(X_{1}, D_{1}\right) \stackrel{\mu_{1}}{\rightarrow} \cdots \stackrel{\mu_{5}}{\rightarrow}\left(X_{6}, D_{6}\right)$. Let $E_{i}$ be the pullbacks of the exceptional curves resulting from blowing up the basepoints of $f$ and $L$ be the pullback of hyperplane sections of $\mathbf{P}^{2}$.

The divisor class groups of the pairs $X_{i}$ for $i \in\{1, \ldots, 6\}$ are generated by $\mathrm{Cl} X_{0}=$ $\mathrm{Cl} X_{1}=\mathrm{Cl} X_{2}=\mathrm{Cl} X_{3}=\mathbf{Z}\left\langle L, E_{1}, \ldots, E_{10}\right\rangle, \mathrm{Cl} X_{4}=\mathrm{Cl} X_{5}=\mathbf{Z}\left\langle L, E_{1}, \ldots, E_{9}\right\rangle$ and $\mathrm{Cl} X_{6}=\mathbf{Z}\left\langle L, E_{1}\right\rangle$, where $L^{2}=1, E_{i} E_{j}=-\delta_{i j}$ and $L E_{i}=0$ for all $i, j \in$ $\{1, \ldots, 10\}$.

Note that $D_{i+1}=\mu_{i *}\left(D_{i}+K_{i}\right)$, and that $\operatorname{dim}\left|D_{6}+K_{6}\right|=-1$ as in Definition 34 . We can determine $D_{0}$ by the basepoint analysis of $f_{0}$. From $K_{\mathbf{P}^{2}}=-3 L$ (see [7]) it follows that the canonical divisor classes $K_{i} \in \mathrm{Cl} X_{i}$ are $K_{i}=-3 L+E_{0}+\cdots+E_{10}$ for $i \in\{0,1,2,3\}, K_{i}=-3 L+E_{0}+\cdots+E_{9}$ for $i \in\{4,5\}$ and $K_{6}=-3 L+E_{1}$.

We represent $D_{i}$ in terms of the generators of $\mathrm{Cl} X_{i}$ for $i \in\{0, \ldots, 6\}$ : 


\begin{tabular}{llllllllllll}
\hline & $L$ & $-E_{1}$ & $-E_{2}$ & $-E_{3}$ & $-E_{4}$ & $-E_{5}$ & $-E_{6}$ & $-E_{7}$ & $-E_{8}$ & $-E_{9}$ & $-E_{10}$ \\
\hline$D_{0}$ & 19 & 7 & 6 & 6 & 6 & 6 & 6 & 6 & 6 & 6 & 4 \\
$D_{1}$ & 16 & 6 & 5 & 5 & 5 & 5 & 5 & 5 & 5 & 5 & 3 \\
$D_{2}$ & 13 & 5 & 4 & 4 & 4 & 4 & 4 & 4 & 4 & 4 & 2 \\
$D_{3}$ & 10 & 4 & 3 & 3 & 3 & 3 & 3 & 3 & 3 & 3 & 1 \\
$D_{4}$ & 7 & 3 & 2 & 2 & 2 & 2 & 2 & 2 & 2 & 2 & - \\
$D_{5}$ & 4 & 2 & 1 & 1 & 1 & 1 & 1 & 1 & 1 & 1 & - \\
$D_{6}$ & 1 & 1 & - & - & - & - & - & - & - & - & - \\
\hline
\end{tabular}

From Theorem 46(a) and $P=\left|L-E_{1}\right|$ in Proposition 44 it follows that $S\left(X_{6}, D_{6}\right)=\{P\}$ and $v\left(X_{6}, D_{6}\right)=0$. From Theorem 46(d) case B3 and then five times B4 it follows that $S\left(X_{0}, D_{0}\right)=\{Q\}$ and $v\left(X_{0}, D_{0}\right)=12$, where $Q=$ $\mu_{0}^{\circledast} \circ \cdots \circ \mu_{5}^{\circledast} P$. In terms of generators of $\mathrm{Cl} X_{0}$ we have $Q=\left|L-E_{1}\right| \in$ Fam $X_{0}$.

We have $g_{0 \circledast} Q=F=\left(F_{i}\right)_{i \in I}$ with $I=\mathbf{P}^{1}$ and $F_{i}=\left\{\left(s: \frac{i_{0}}{i_{1}} s: u\right)\right\}$ for $i=$ $\left(i_{0}: i_{1}\right) \in I$. After dehomogenization of $F$ to $s$ and $i_{1}$ we have $I=\mathbf{C}$ and $F_{i}=$ $\{(1: i: u) \mid u \in \mathbf{C}\}^{-}$for $i \in I$.

From Proposition 50 it follows that $S\left(Y_{0}\right)=\left\{\varphi_{D_{0} \circledast}(Q)\right\}$ and $v\left(Y_{0}\right)=v\left(X_{0}, D_{0}\right)$ where

$$
\varphi_{D_{0} \circledast}(Q)=\varphi_{D_{0} \circledast}\left(g_{0}^{\circledast}(F)\right)=\left\{f_{0}(1: i: u) \mid u \in \mathbf{C}\right\}_{i \in I} .
$$

The degree of this family is $\operatorname{deg}_{u} f_{0}(1: i: u)=12$. Indeed this is equal to $v\left(X_{0}, D_{0}\right)=12$.

It is remarkable that on a rational surface of degree 8 the optimal family has degree 12 .

Acknowledgements This research was supported by the Austrian Science Fund (FWF) in the frame of the research project P-21461.

\section{References}

1. Cox, D.: What is a toric variety. In: Topics in Algebraic Geometry and Geometric Modeling. Contemp. Math., vol. 334, pp. 203-223. Am. Math. Soc., Providence (2003)

2. Draisma, J., McAllister, T.B., Nill B.: Lattice width directions and Minkowski's $3^{d}$-theorem. Technical Report, arXiv:0901.1375v1 [math.CO] (2009)

3. Günter, Ewald: Combinatorial Convexity and Algebraic Geometry. Graduate Texts in Mathematics, vol. 168. Springer, New York (1996). ISBN 0-387-94755-8

4. Fulton, W.: Introduction to Toric Varieties. Annals of Mathematics Studies, vol. 131. Princeton University Press, Princeton (1993)

5. Haase, C., Schicho, J.: Lattice polygons and the number $2 i+7$. Math. Mon. (2009)

6. Halphen, G.H.: On plane curves of degree six through nine double points (in french). Bull. Soc. Math. Fr. 10, 162-172 (1882)

7. Hartshorne, R.: Algebraic Geometry. Graduate Texts in Mathematics, vol. 52. Springer, New York (1977). 
8. Manin, Ju.I.: Rational surfaces over perfect fields. Publ. Math. Inst. Hautes Etudes Sci. 30, 55-113 (1966)

9. Matsuki, K.: Introduction to the Mori Program. Universitext. Springer, New York (2002)

10. Schicho, J.: Rational parametrization of surfaces. J. Symb. Comput. 26(1), 1-30 (1998)

11. Schicho, J.: The multiple conical surfaces. Beitrage Algebra Geom. 42, 71-87 (2001)

12. Schicho, J.: Simplification of surface parameterizations-a lattice polygon approach. J. Symb. Comput. 36, 535-554 (2003) 